\title{
Inclusion Complexes of Chain Molecules with Cycloamyloses III. Molecular Dynamics Simulations of Polyrotaxanes Formed by Poly(propylene glycol) and $\beta$-Cyclodextrins
}

\author{
Javier Pozuelo, Francisco Mendicuti, and Wayne L. Mattice* \\ Departamento de Química Física, Universidad de Alcalá, Alcalá de Henares, Madrid, Spain \\ * Institute of Polymer Science, The University of Akron, Akron, OH 44325-3909, U.S.A.
}

(Received October 27, 1997)

\begin{abstract}
Molecular dynamics simulations were performed in vacuo on "channel type" polyrotaxanes composed of $\beta$-cyclodextrins ( $\beta \mathrm{CDs}$ ) threaded onto isotactic and syndiotactic poly(propylene glycol) (PPG). In the most stable complex, the $\beta$ CDs form a close-packed structure from one end of the PPG chain to the other. Non-bonded van der Waals interactions between $\beta C D$ and PPG are the main source of stabilization of the complex. Head-to-head and tail-to-tail orientation of successive $\beta C D$ s in the complex is more favorable than a head-to-tail orientation, due to intermolecular hydrogen bonding between head-to-head $\beta C D$ units. $\beta C D$ s in polyrotaxanes adopt a more rigid and symmetrical macroring conformation than does an isolated $\beta C D$. Formation of the polyrotaxane is accompanied by an increase in the number of trans states at the bonds in the backbone of PPG. For this reason, the PPG chain in the polyrotaxane is much more extended than the unperturbed chain.
\end{abstract}

KEY WORDS Cyclodextrin / Inclusion Complex / Poly(propylene glycol) / Polyrotaxane /

Cyclodextrins (CDs) are cyclic oligosaccharides formed by $6(\alpha \mathrm{CD}), 7(\beta \mathrm{CD}), 8(\gamma \mathrm{CD})$, or $9(\delta \mathrm{CD})$ glucopyranose units connected by $\alpha-1,4$-linkages. The CDs form inclusion complexes with many compounds of low molecular weight. ${ }^{1-3}$ Inclusion complexes of CDs and several polymers have also been prepared. ${ }^{4-11}$ These complexes, included in a group named "polyrotaxanes," are comprised of macrocycles threaded by linear polymer chains, with no covalent bonding between the cyclic and the linear components. These molecular assemblies are important in supramolecular chemistry. Potential areas of utilization include improved interfacial bonding (adhesion, blend compatibilization), processability (enhanced solubility), improved control of thermal properties (glass transition, melting temperatures), viscosity, and molecular ordering.

Harada et al. ${ }^{4-11}$ have prepared and characterized "channel type" ${ }^{12}$ polyrotaxanes that form spontaneously from several polymers and CDs in aqueous solution. In general, a very selective formation of polyrotaxanes was observed. The rate and the yield of the complex formation depends strongly on the nature and molecular weight $(M)$ of the polymer. The relative sizes of the cavities of the CDs and the cross sectional areas of the polymer are important in complex formation.

The $\alpha \mathrm{CD}$ forms complexes with poly(ethylene glycol) (PEG) chains. ${ }^{5,6,8}$ In contrast, $\beta C D$ does not form complexes with PEG of any $\mathrm{M} .^{4}$ Some PEG derivatives containing bulky groups at the end of the chain can be included to form high yields of crystalline complexes with $\gamma$ CD. ${ }^{9} \alpha \mathrm{CD}$ does not form complexes with these PEG derivatives when the end groups are too large. There are one (or two) chains of PEG threaded through $\alpha \mathrm{CD}$ (or $\gamma \mathrm{CD}$ ) in the complexes. A polyrotaxane was also synthesized from monodisperse PEG (M 1248) and $\alpha \mathrm{CD}$, with the $\mathrm{CD}$ units imprisoned by capping the ends of the chain with bulky substituents.

Poly (propylene glycol) (PPG) chains of various $M$ were also used to form polyrotaxanes with $\beta$ - and $\gamma \mathrm{CDs}$ in high yields, although this polymer does not form complexes with lower molecular weight analogues.,7,11 Harada et al. ${ }^{11}$ have studied the complex of PPG and $\beta C D$ in detail. $\beta C D$ forms crystalline complexes with PPG of $M>400$. These complexes are stable without being end-capped. Yields of the complexes rise with an increase in $M$ of the chain in the range 400-1000, but yields decrease when $M$ of the PPG becomes larger than 1000. Continuous variation plots for the formation of the complexes of PPG ( $M$ 1000) and $\beta C D$ suggest that a CD interacts with two repeat units of the PPG chain. ${ }^{1} \mathrm{H}$ NMR supports this conclusion. Differential thermal analysis shows that complexation stabilizes $\beta \mathrm{CD}$. An $X$-ray study of a single crystal of the complex shows that the PPG : $\beta C D$ complex has a "channel type" structure. CP/MAS NMR and X-ray studies show that $\beta C D$ adopts a more symmetrical conformation in the complex than when it does not include a guest in its cavity. X-ray studies on a single crystal of the complex between $\beta C D$ and $p$-nitroacetanilide prove that head-to-head, tailto-tail orientations result in an effective formation of hydrogen bonds between CDs, and this orientation is thought to be the most probable mode of CD packing. Isotactic PPG chains form complexes with $\beta C D$ in lower yield than do atactic PPG chains of similar average $M$. This fact has been rationalized by invoking a larger effective cross-section for isotactic PPG than atactic PPG, which impedes the movement of the chain into the cavity of $\beta \mathrm{CD}$.

Molecular dynamics (MD) simulations have been used to study the conformations and mobility of similar systems in which a polymer is confined in a narrow channel, ${ }^{13-18}$ including MD simulations ${ }^{19}$ of complexes of $\alpha \mathrm{CD}$ with end-capped PEG of the kind described by Harada, ${ }^{10}$ favoring $2.6( \pm 0.1)$ oxyethylene units per $\mathrm{CD}$. The $\alpha \mathrm{CDs}$ in the complex formed a close-packed structure from one end of the PEG chain to the other end. The van der Waals non-bonded interactions provide the main source of stability for the 
complex. Hydrogen bonds favor slightly head-to-head, tail-to-tail sequences of $\alpha \mathrm{CD}$. The $\alpha \mathrm{CD}$ in the complex is more symmetric than the isolated $\alpha \mathrm{CD}$, and the PEG in the complex is more extended than the unperturbed chain.

Here MD simulations are used to study polyrotaxanes of PPG chains and $\beta \mathrm{CD}$.

\section{METHODOLOGY FOR THE SIMULATIONS}

The MD trajectories were computed using Sybyl 6.3 from Tripos Asociates (St. Louis, Missouri) and the Tripos Force Field 5.2. ${ }^{20}$ The contribution to the energy from the hydrogen bonds in incorporated in the van der Waals and coulombic terms. All simulations were performed in vacuo, rather than in aqueous solution, because of the prohibitive amount of computer time required for simulations in the dense liquid. The molecules studied were isolated chains of the isotactic poly$((R)$-propylene glycol) and the syndiotactic poly $((R S)$ propylene glycol) containing ten monomer units and the complexes of these chains with $\beta C D$. The isolated chains are abbreviated as PPG $n$ t, where $n$ denotes the number of monomer units and $t$ is the tacticity of the chain, denoted by i for isotactic and by s for syndiotactic. Polyrotaxanes are abbreviated as RPPGntCDm(SE), where $m$ denotes the number of molecules of $\beta C D$ in the complex, and SE denotes their sequence, using $\mathrm{HH}$ for head-to-head, tail-to-tail and HT for head-to-tail. The polyrotaxanes of PPG with $\beta C D$ that were synthesized by the Harada group do not need to be end capped by bulky groups,${ }^{11}$ perhaps because PPG is more hydrophobic than PEG. Nevertheless, for our simulations in vacuo, the polyrotaxanes as well as the free PPG chains were capped at both ends with 9-anthracenamine to avoid dethreading during the simulation. Figure 1 depicts isolated PPGni and PPGns, as well as the polyrotaxanes named RPPGniCD $m \mathrm{HT}$ and RPPG $n \mathrm{SCD}$ $m \mathrm{HH}$.

The potential energy of each image is evaluated as a sum of six contributions: bond stretching, bond angle bending, torsion, out of plane, van der Waals and electrostatic interactions. Typically the contribution to the binding energy from the electrostatic energy is only around $1 \%$ of the contribution from the van der Waals interactions. Therefore the simulation is insensitive to the details of the treatment of the electrostatic interactions. Following Brant and flory, ${ }^{21-23}$ electrostatic interactions were taken into account using pairwise contributions of $q_{i} q_{j} / 3.5 r_{i j}$, where $r_{i j}$ is the separation of partial charges $i$ and $j$. Geometry and partial charges for PPG10i, PPG10s, and $\beta C D$ as isolated molecules and polyrotaxanes were obtained by MOPAC. ${ }^{24}$ A tetramer of PPG, capped with 9-anthracenamine at both ends, was used to calculate the geometry and the partial charges for PPGs. Geometry and charges for $\beta \mathrm{CD}$ are the ones used previously. ${ }^{25}$

The starting conformation had trans states at all of the internal bonds in the PPG. The $\beta$ CDs were initially in the nondistorted conformation described previously, with $\phi$ and $\psi$, the two torsions at the bonds to the bridging oxygen atom, at $0^{\circ}$ and $-3^{\circ}$, respectively, and the bond angle at this atom, $\tau$, at $121.7^{\circ} .{ }^{19,25}$ Starting conforma-

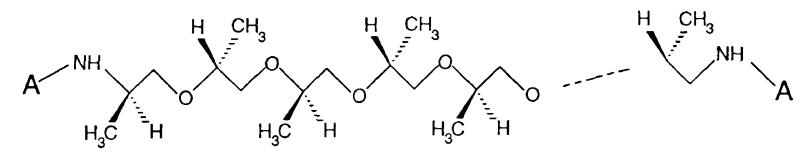

PPGni

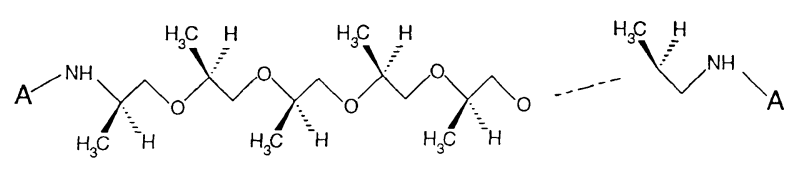

PPGns

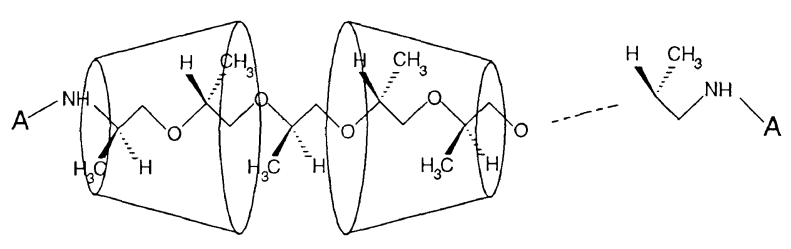

RPPGniCDmHH

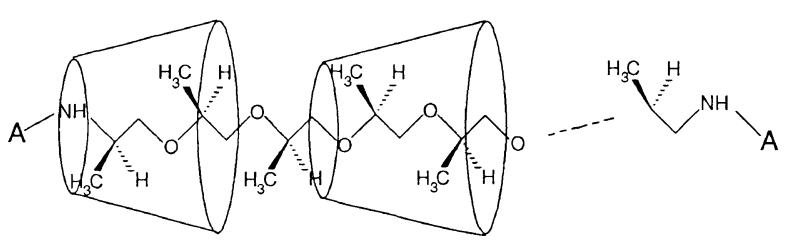

RPPGnsCDmHT

Figure 1. Isolated PPGni and PPGns, as well as the polyrotaxanes named RPPG $n$ iCDmHT and RPPG $n \mathrm{CD} m \mathrm{HH}$.

tions for the polyrotaxanes were constructed from $m$ nondistorted $\beta \mathrm{CDs}$, with centers separated by $8 \AA$, around the extended PPG chain.

Calculations were performed in a canonical ensemble, with $N V T$ fixed. Lengths were constrained for bonds to hydrogen atoms, but all other bond lengths, bond angles and torsional angles were variable during the simulations. From $0 \mathrm{~K}, T$ was increased $10 \mathrm{~K}$ at intervals of $300 \mathrm{fs}$, and the molecule was equilibrated at the final $T(500 \mathrm{~K})$ for up to $100 \mathrm{ps}$ before the collection of data was initiated. The momentum reset and the coupling factor were $10 \mathrm{fs}$. Velocities were rescaled at intervals of $10 \mathrm{fs}$. The duration of the trajectory was $0.5 \mathrm{~ns}$, computed with a time step of $2 \mathrm{fs}$. Conformations were saved at intervals of $200 \mathrm{fs}$, yielding 2500 images from each simulation for subsequent analysis. The average of any property $\langle X\rangle$ was obtained as

$$
\langle X\rangle=\frac{1}{N} \sum X_{i}
$$

where $N$ is the number of images and $X_{i}$ is the value of the property for image $i$.

For each image of the polyrotaxanes, the non-bonded interaction between the PPG chain and CDs, denoted $E_{\text {binding, }}$, was evaluated as the difference between the total potential energy and the potential energy of the PPG chain and $m$ CD units. 


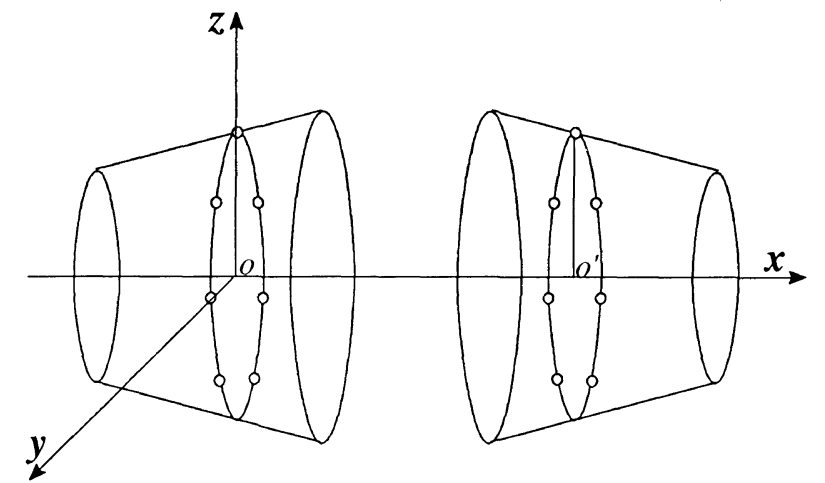

Figure 2. Scheme for defining the distance between two $\beta \mathrm{CDs}$, using the points $O$ and $O^{\prime}$.

$$
E_{\text {binding }}=E_{\mathrm{RPPG} n \mathrm{tCDm}}-\left(E_{\mathrm{PPG} n \mathrm{t}}+E_{\mathrm{CDm}}\right)
$$

The term $E_{\mathrm{RPPG} \text { tCDm }}$ represents the total energy of the system, and $E_{\mathrm{PPG} n}$ and $E_{\mathrm{CDm}}$ are the potential energy of the system taking into account only the atoms of PPG or $m$ CDs, respectively.

As previously, ${ }^{19}$ for evaluating the number of intraand intermolecular hydrogen bonds of CDs, a hydrogen bond is assumed when the $\mathrm{O} \cdots \mathrm{H}$ distance is 0.8 $2.8 \AA$ and the angle formed by $\mathrm{O} \cdots \mathrm{H}-\mathrm{O}$ is in the range $120-180^{\circ}$

\section{RESULTS AND DISCUSSION}

Study of the PPG: $\beta C D$ Ratio in the Rotaxane

A chain-independent estimate of the amount of $\beta C D$ that might be bound to a linear chain can be obtained by considering the nonbonded binding energy (calculated in a manner similar to eq 2 ) between two isolated $\beta C D$ s, as a function of the distance between them. The two CDs approach one another, with a head-to-head orientation, along a virtual $x$ axis that passes through the centroids $O$ and $O^{\prime}$ defined by the seven bridging oxygen atoms of each CD, as depicted in Figure 2. Figure 3 depicts the results obtained by two methods, the one using a molecular mechanics calculation and the other using a molecular dynamics calculation. The most negative $E_{\text {binding }}$ occurs when the separation of the $\beta C D$ s in near $8 \AA$. A strongly repulsive interaction is generated if the separation is reduced to $7 \AA$.

Specialization of these results to a particular polymer requires an estimate of the extension of its monomer unit. The maximum extension of a monomer unit of PEG or PPG, while retaining the preferred bond lengths and bond angles, is slightly more than $3 \AA$. The vertical dashed lines in Figure 3 denote the distance between CDs for two different PPG: $\beta C D$ ratios, assuming this extension for the polymer. According to these results, a polyrotaxane with a composition of two PPG units per $\beta C D$ is less likely than one with a composition closer to $2.5 \mathrm{PPG}$ units per $\beta \mathrm{CD}$. This result is somewhat in conflict with the report by Harada et al. ${ }^{11}$ of a ratio of two oxypropylene units per $\beta C D$ in the polyrotaxane. They earlier reported results for end-capped polyrotaxanes prepared from monodisperse PEG and $\alpha \mathrm{CD}$ where there were 2.3 ethylene glycol units per CD, ${ }^{8,10}$ which is a composition closer to the expectation from

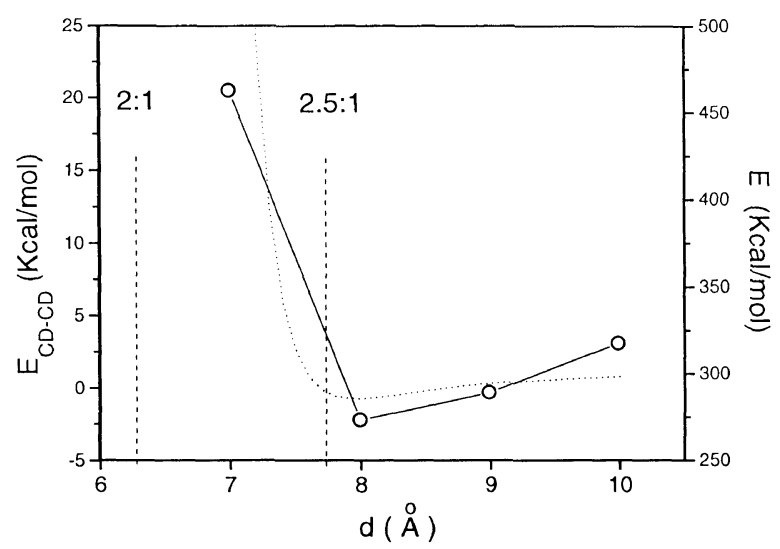

Figure 3. Binding energy $(O)$ and total potential energy $(\cdots)$ as a function of separation for two head-to-head $\beta C D$ s. The four points were obtained by MD, and the curved dotted line is from MM. The two vertical dashed lines denote the separations for 2 and 2.5 extended PPG (or PEG) units per $\beta C D$.

Table I. Binding energies and its components ( $\mathrm{kcal} \mathrm{mol}^{-1}$ ) for four polyrotaxanes

\begin{tabular}{lccc}
\hline Polyrotaxane & $\begin{array}{c}\text { Binding } \\
\text { energy }\end{array}$ & $\begin{array}{c}\text { van der Waals } \\
\text { part }\end{array}$ & $\begin{array}{c}\text { Electrostatic } \\
\text { part }\end{array}$ \\
\hline RPPG10iCD4HH & -88.0 & -87.3 & -0.8 \\
RPPG10sCD4HH & -86.7 & -86.0 & -0.8 \\
RPPG10iCD4HT & -88.4 & -87.4 & -1.0 \\
RPPG10sCD4HT & -86.8 & -86.0 & -0.9 \\
\hline
\end{tabular}

Figure 3. Perhaps the difference in composition arises from the absence of end caps in their polyrotaxane of PPG and $\beta C D$. The PPG used in the experiment had about 17 monomer units per chain. This chain would thread 6.8 or $8.5 \mathrm{CDs}$ for compositions of $2.5: 1$ and $2: 1$, respectively. These two numbers of CDs differ by less than 2, suggesting that the slightly higher than expected (from Figure 3) number of $\beta C D$ s per chain might be attributed to incomplete threading of a CD at each of the uncapped ends of the PPG used in the experiment.

\section{Stabilization of the Polyrotaxanes}

The $E_{\text {binding }}$ and its components are collected in Table I for the polyrotaxanes. All four polyrotaxanes have negative values of $E_{\text {binding. The van der Waals interac- }}$ tions contribute about $99 \%$ of the stabilization of the complex. Polyrotaxanes formed by isotactic PPG are $1-2 \mathrm{kcal} \mathrm{mol}^{-1}$ more stable than the polyrotaxanes formed by syndiotactic PPG. A further breakdown of the components of the energies, not included in Table I, shows that the strain energy $\left(E_{\text {stretching }}+E_{\text {binding }}+\right.$ $\left.E_{\text {torsional }}\right)$ favors the HT orientation of the $\beta \mathrm{CDs}$, but the van der Waals interactions favor the $\mathrm{HH}$ orientation, with the net result being virtually no discrimination between the two orientations for the polyrotaxanes.

\section{Intra- and Intermolecular Hydrogen Bonding Interactions of $\beta C D s$ in the Polyrotaxanes}

As shown in Table II, nearly identical averages of more than two intramolecular hydrogen bonds per $\beta C D$ unit are obtained during each simulation. The intermolecular hydrogen bonds between $\beta \mathrm{CDs}$, in contrast, depend on 
Table II. Average number of hydrogen bonds in four polyrotaxanes ${ }^{\mathrm{a}}$

\begin{tabular}{|c|c|c|c|c|}
\hline Type & RPPG10iCD4HH & RPPG10sCD4HH & RPPG10iCD4HT & RPPG10sCD4HT \\
\hline Intra $\beta C D$ & $8.6 \pm 2.6$ & $8.7 \pm 2.6$ & $8.8 \pm 2.7$ & $9.0 \pm 2.7$ \\
\hline$\beta \mathrm{CD} 1$ & $2.2 \pm 1.3$ & $2.3 \pm 1.4$ & $2.3 \pm 1.4$ & $2.2 \pm 1.3$ \\
\hline$\beta \mathrm{CD} 3$ & $2.1 \pm 1.3$ & $2.2 \pm 1.3$ & $2.1 \pm 1.4$ & $2.3 \pm 1.4$ \\
\hline$\beta \mathrm{CD} 4$ & $2.2 \pm 1.3$ & $2.0 \pm 1.3$ & $2.1 \pm 1.3$ & $2.3 \pm 1.4$ \\
\hline Inter $\beta \mathrm{CDs}$ & $5.7 \pm 2.5$ & $5.9 \pm 2.5$ & $3.7 \pm 1.9$ & $3.6 \pm 1.9$ \\
\hline$\beta \mathrm{CD} 1 \beta \mathrm{CD} 2$ & $2.6 \pm 1.6$ & $2.8 \pm 1.8$ & $1.2 \pm 1.1$ & $1.2 \pm 1.1$ \\
\hline$\beta \mathrm{CD} 2 \beta \mathrm{CD} 3$ & $0.6 \pm 0.8$ & $0.7 \pm 0.7$ & $1.2 \pm 1.1$ & $1.2 \pm 1.1$ \\
\hline$\beta \mathrm{CD} 3 \beta \mathrm{CD} 4$ & $2.5 \pm 1.7$ & $2.4 \pm 1.7$ & $1.3 \pm 1.1$ & $1.1 \pm 1.1$ \\
\hline$\beta \mathrm{CD}-\mathrm{PPG}$ & $0.6 \pm 0.7$ & $0.5 \pm 0.7$ & $0.6 \pm 0.7$ & $0.4 \pm 0.7$ \\
\hline$\beta \mathrm{CD}$-Ends & $0.2 \pm 0.5$ & $0.2 \pm 0.4$ & $0.2 \pm 0.5$ & $0.0 \pm 0.0$ \\
\hline
\end{tabular}

${ }^{a}$ Line 1: Average number of intramolecular hydrogen bonds in the $\beta \mathrm{CD}$; Lines $2-5$ : Contribution by each $\beta \mathrm{CD}$ to the total number of intramolecular hydrogen bonds; Line 6: Average number of intermolecular hydrogen bonds between $\beta$ CDs; Lines $7-9$ : Contribution by each pair of $\beta \mathrm{CDs}$ to the intermolecular hydrogen bonds; Lines 10: Average number of hydrogen bonds between $\beta$ CDs and PPT; Lines 11: Average number of hydrogen bonds between $\beta C D$ s and the end groups.

Table III. Population of the trans state at the bonds in the polymer

\begin{tabular}{lccc}
\hline \multicolumn{1}{c}{ Molecule } & $\mathrm{CH}\left(\mathrm{CH}_{3}\right)-\mathrm{CH}_{2}$ & $\mathrm{CH}_{2}-\mathrm{O}$ & $\mathrm{O}-\mathrm{CH}\left(\mathrm{CH}_{2}\right)$ \\
\hline PPG $\infty \mathrm{i}^{\mathrm{a}}$ & 0.34 & 0.86 & 0.54 \\
$\mathrm{PPG} \propto \mathrm{s}^{\mathrm{a}}$ & 0.33 & 0.92 & 0.58 \\
$\mathrm{RPPG10iCD4HH}$ & 0.92 & 0.91 & 0.71 \\
$\mathrm{RPPG10sCD4HH}$ & 0.91 & 0.94 & 0.76 \\
RPPG10iCD4HT & 0.93 & 0.92 & 0.74 \\
RPPG10sCD4HT & 0.94 & 0.93 & 0.82 \\
\hline
\end{tabular}

a Calculated at $500 \mathrm{~K}$, using rotational isomeric state "Model I" of Abe et al. ${ }^{26}$

the sequence of the $\beta C D$ s. In support of the conclusion from Harada's group, ${ }^{6,11}$ intermolecular hydrogen bonds between $\beta \mathrm{CDs}$ are more numerous for $\mathrm{HH}$ sequences than for HT ones. The larger contribution to the total intermolecular hydrogen bonds between pairs of $\beta \mathrm{CDs}$ for $\mathrm{HH}$ sequences comes from the head-to-head interactions of the first and last pair of $\beta C D$ s. Tacticity of the PPG has little influence on the number of hydrogen bonds between the $\beta C D$ s. The hydrogen bonds between the $\beta C D$ and the PPG or its bulky end groups are less important than the hydrogen bonds within and between CDs.

\section{Conformation Analysis of PPG in the Complex}

The internal bonds in PPG prefer trans, gauche ${ }^{+}$, and gauche states. $^{26}$ The populations of the trans state at the three types of internal bonds in the chains in the polyrotaxanes in the simulations are compared in Table III with those extracted from the rotational isomeric state model devised for unperturbed PPG by Abe et al. ${ }^{26}$ All of the bonds have a higher population of trans states in the polyrotaxanes than in the unperturbed chain. As a consequence, the PPG chains in the polyrotaxanes are more extended than the unperturbed chains. The magnitude of the change in extension is apparent upon examination of the dimensionless characteristic ratio,

$$
C_{n} \equiv \frac{\left\langle r^{2}\right\rangle}{\sum_{i=1}^{n} l_{i}^{2}}
$$

which is the ratio of the mean square end-to-end distance, $\left\langle r^{2}\right\rangle$, and the sum of the squares of the lengths of the $n$ bonds in the chain. For the rotational isomeric state model described by Abe et al. for unperturbed isotactic and syndiotactic PPG, $C_{n}$ is in the range 4.6-4.9 at the degree of polymerization and temperature of the present simulation. ${ }^{26}$ In contrast, the characteristic ratios for the PPG chains in the four polyrotaxanes are in the range 17.1-17.4, which is more than three times as large as the $C_{n}$ for the unperturbed chains. Nevertheless, the characteristic ratios for the PPG chains in these polyrotaxanes with $\beta C D$ are not quite as large as those determined earlier for PEG chains in polyrotaxanes with $\alpha$ CD. $^{19}$

\section{Conformation Analysis of the $\beta C D$ s in the Complex}

The distribution function for the torsion angles at the $\mathrm{C}-\mathrm{O}$ bonds to the bridging oxygen atom, $\phi$ and $\psi$, are qualitatively the same at the $\beta C D$ s in all four polyrotaxanes. The distributions exhibit two maxima located roughly $30^{\circ}$ on either side of the center of the trans state $\left(0^{\circ}\right)$, as illustrated in Figure 4 . In contrast, the distributions for isolated $\beta \mathrm{CD}$ show two well-defined regions at approximately $\pm 60^{\circ}$ on either side of $0^{\circ}$, as well as occasional occupation of the cis state at $\psi .^{22}$ Formation of the complex reduces the internal conformational freedom of $\beta C D$. In its complex with PEG, $\alpha \mathrm{CD}$ shows a stronger constraint on the distribution of torsion angles at $\phi$ and $\psi$, with the appearance of a single maximum. ${ }^{19}$ Thus the $\beta C D$ in the polyrotaxane with PPG has more internal conformational freedom than does the $\alpha \mathrm{CD}$ in its polyrotaxane with PEG.

The values of the bond angles $\tau$, defined by $\mathrm{C}(1)$ $\mathrm{O}-\mathrm{C}\left(4^{\prime}\right)$, are similar for all $\beta \mathrm{CDs}$. The average value, $117.2 \pm 0.1^{\circ}$, is close to the result of $117.3^{\circ}$ for the isolated $\beta \mathrm{CD}$, and far from the value of $121.7^{\circ}$ in the initial conformation of the polyrotaxane.

The alteration in the distributions for $\phi$ and $\psi$ upon formation of the complex implies changes in the shape and size of the cavity in $\beta C D$. The size and shape of this cavity is described, as previously, ${ }^{19,22}$ using parameters that define a torus, as depicted in Figure 5. The larger radius, $R$, is the average of the distance of the 14 oxygen atoms in all secondary hydroxyl groups from their center of mass. The smaller radius, $r$, is defined in a similar manner, using the seven oxygen atoms in the 

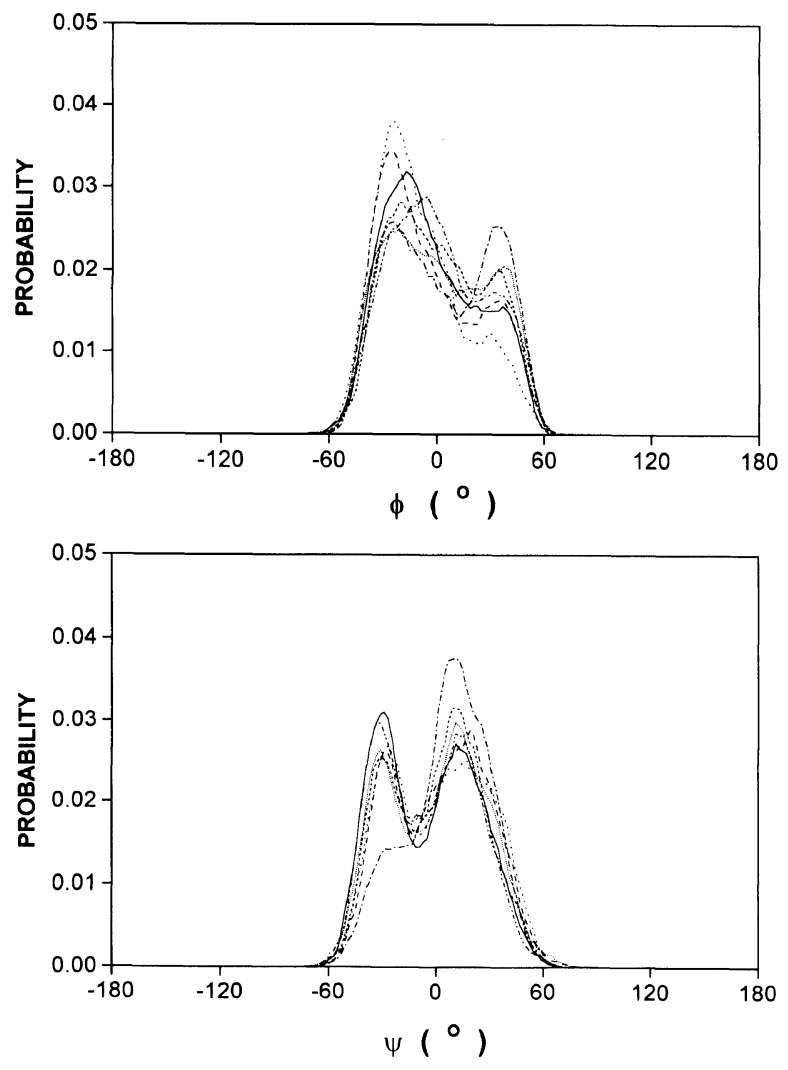

Figure 4. Probability distributions for the torsion angles at the bonds to the bridging oxygen atoms in seven $\beta$ CDs in RPPG10iCD4HT. $(-) 1,(---) 2,(\cdots) 3,(-\cdot-) 4,(-\cdot \cdot) 5,(--) 6,(\cdots) 7$.

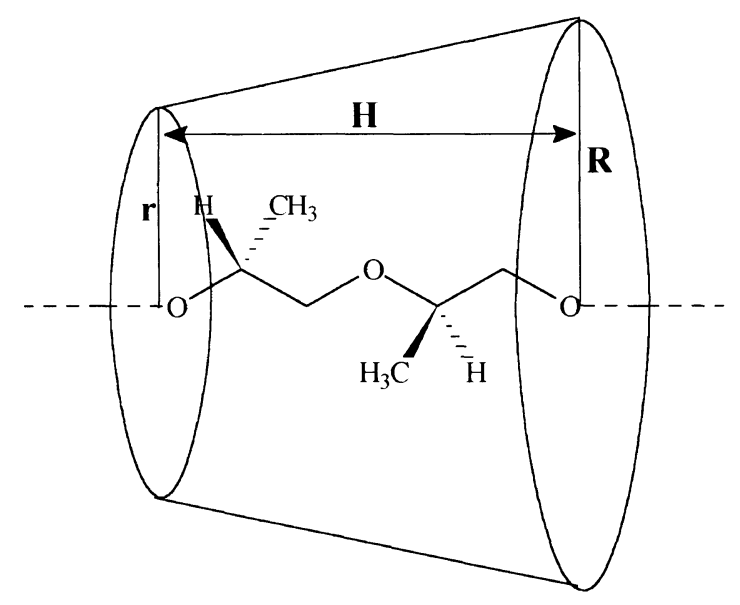

Figure 5. Schematic of $R, r$, and $H$ used to define the torus that describes the cavity in $\beta C D$. primary hydroxyl groups. The height $H$ is the distance between the centers of mass defined by the oxygen atoms in the two types of hydroxyl groups. A subscript $t$ appended to these parameters denotes the subtraction (for $R_{\mathrm{t}}$ and $r_{\mathrm{t}}$ ) of the van der Waals radius $(1.36 \AA$ ) of an oxygen atom, and for $H_{\mathrm{t}}$ it denotes the addition of the van der Waals diameter of an oxygen atom. The volume of the torus is

$$
V_{\mathrm{t}}=\pi H_{\mathrm{t}}\left[r_{\mathrm{t}} R_{\mathrm{t}}+\frac{\left(R_{\mathrm{t}}^{2}-r_{\mathrm{t}}^{2}\right)}{3}\right]
$$

The mean squared radii of gyration, $\left\langle s^{2}\right\rangle$, were also calculated for three subsets of oxygen atoms. These subsets are denoted by "ring" for the seven briding oxygen atoms, "tail" for the seven oxygen atoms bonded to C6, and "head" for the 14 oxygen atoms in the secondary hydroxyl groups.

By all of these measures, the $\beta C D$ s have similar shapes in all four of the polyrotaxanes, as shown in Table IV. This shape is induced by the formation of the complex, because the isolated $\beta C D$ has distinctly different values for most of these parameters. ${ }^{22}$ The isolated $\beta C D$ has a cavity with a smaller volume, $368(32) \AA^{3}$, arising from a smaller height, $6.10(0.34) \AA$, and smaller $\left\langle r_{\mathrm{t}}\right\rangle, 3.78$ (0.36) $\AA$. The torus becomes more symmetric in shape upon formation of the polyrotaxane, as judged by the size of $\left\langle r_{\mathrm{t}}\right\rangle /\left\langle R_{\mathrm{t}}\right\rangle$, which is 0.76 for the isolated $\beta \mathrm{CD}$, but in the range $1.00-1.06$ for the $\beta C D$ s in the polyrotaxanes. The shape changes from a cone in the isolated $\beta C D$ to a cylinder for the $\beta C D$ in the complex, as was observed previously for $\alpha \mathrm{CD}$ and its complex with PEG. ${ }^{19}$ The isolated $\beta \mathrm{CD}$ has smaller radii of gyration denoted by $\left\langle s^{2}\right\rangle_{\text {ring }}^{1 / 2}, 5.0 \AA$, and $\left\langle s^{2}\right\rangle_{\text {tail }}^{1 / 2}, 5.5 \AA$, but a slightly larger $\left\langle s^{2}\right\rangle_{\text {head }}^{1 / 2}, 6.5 \AA$. These three radii of gyration differ more strongly from one another in the isolated $\beta C D$ than for the $\beta C D$ s in the polyrotaxanes, as a consequence of the more symmetric shape of the $\beta C D$ s in the complex. Standard deviations for $\left\langle s^{2}\right\rangle_{\text {ring }}^{1 / 2}$ are about three times larger for the isolated $\beta C D$ than for the $\beta C D$ s in the polyrotaxanes, which documents the reduction in flexibility of the ring when the $\beta \mathrm{CD}$ forms the complex. In comparison, there is little change in the standard deviations for $\left\langle s^{2}\right\rangle_{\text {tail }}^{1 / 2}$ and $\left\langle s^{2}\right\rangle_{\text {head }}^{1 / 2}$ upon formation of the complex.

\section{CONCLUSIONS}

Analysis of the MD simulations in vacuo for PPG: $\beta C D$ polyrotaxanes suggests a composition of about 2.5 monomer units per $\mathrm{CD}$, with $\beta \mathrm{CD}$ separated by approximately $8 \AA$, forming a close-packed structure

Table IV. Size and shape of the cavity in the $\beta C D$ s in the four polyrotaxanes Distances in $\AA$, volumes in $\AA^{3}$, standard deviation in parenthesis.

\begin{tabular}{|c|c|c|c|c|}
\hline Parameter & RPPG10iCD4HH & RPPG10sCD4HH & RPPG10iCD4HT & RPPG10sCD4HT \\
\hline$\left\langle H_{\mathrm{t}}\right\rangle$ & $7.85(0.23)$ & $7.97(0.23)$ & $7.86(0.26)$ & $7.90(0.22)$ \\
\hline$\left\langle R_{\mathrm{t}}\right\rangle$ & $4.61(0.21)$ & $4.64(0.21)$ & $4.65(0.21)$ & $4.68(0.21)$ \\
\hline$\left\langle r_{\mathrm{t}}\right\rangle$ & $4.90(0.37)$ & $4.72(0.36)$ & $4.66(0.35)$ & $4.69(0.34)$ \\
\hline$\left\langle V_{\mathrm{t}}\right\rangle$ & $559(37)$ & $548(35)$ & $535(31)$ & $545(33)$ \\
\hline$\left\langle s^{2}\right\rangle_{\text {ring }}^{1 / 2}$ & $5.6(0.06)$ & $5.6(0.06)$ & $5.6(0.06)$ & $5.6(0.07)$ \\
\hline$\left\langle s^{2}\right\rangle_{\text {head }}^{1 / 2}$ & $6.1(0.21)$ & $6.1(0.20)$ & $6.1(0.20)$ & $6.1(0.20)$ \\
\hline$\left\langle s^{2}\right\rangle_{\text {tail }}^{1 / 2}$ & $6.4(0.35)$ & $6.2(0.35)$ & $6.2(0.35)$ & $6.2(0.34)$ \\
\hline
\end{tabular}


from one end to the other. As was the case with PEG : $\alpha \mathrm{CD}$ complexes, ${ }^{19}$ van der Waals interaction between PPG and $\beta C D$ is the main contribution to the stabilization of the polyrotaxanes. Head-to-head, tail-to-tail sequences of $\beta C D$ s are more stable than head-to-tail sequences in the polyrotaxanes, due to intermolecular hydrogen bonding interactions between $\beta C D$, where the large contribution is due to intermolecular hydrogen bonds at the head-to-head junctions. $\beta C D$ s in polyrotaxanes have a cylindrical shape, and they are less distorted than the isolated $\beta C D$. Their macrorings are more symmetric and less flexible in the complex than in the isolated $\beta C D$. However, the tails and heads which define the entrance into the cavity of the $\beta C D$ in the complex have a flexibility similar to that seen in the isolated $\beta C D$. A twist around the trans states at the bonds to the bridging oxygen atoms is observed for $\beta C D$ s in the complex, and the cis state is total suppressed. The trans states are strongly preferred at all internal bonds of the PPG chains in the complex, causing them to become much more extended than in the unperturbed state. The calculated binding energy suggests complexes formed with isotactic PPG chains are slightly more stable than those formed with syndiotactic ones.

Acknowledgments. This work was supported by DGICYT PG94-0364 and by National Science Foundation grant DMR 9523278.

\section{REFERENCES}

1. M. L. Bender and M. Komillama, "Cyclodextrins Chemistry," Springer Verlag, Berlin, 1978.
2. J. Szejtli, "Cyclodextrin and Their Inclusion Complexes," Akadémiai Kiadó, Budapest, 1982.

3. J. Szejtli, "Cyclodextrins Technology," Kluver Academic Publishers, Dordrecht, 1988.

4. A. Harada and M. Kamachi, J. Chem. Soc., Chem. Commun., $1322(1990)$

5. A. Harada and M. Kamachi, Macromolecules, 23, 2823 (1990).

6. A. Harada, J. Li, and M. Kamachi, Nature, 356, 325 (1992).

7. A. Harada, J. Li, and M. Kamachi, Macromolecules, 26, 5698 (1993).

8. A. Harada, J. Li, T. Nakamitsu, and M. Kamachi, J. Org. Chem., 58, 7524 (1993).

9. A. Harada, J. Li, and M. Kamachi, Nature, 370, 126 (1994).

10. A. Harada, J. Li, and M. Kamachi, J. Am. Chem. Soc., 116, 3192 (1994).

11. A. Harada, M. Okada, J. Li, and M. Kamachi, Macromolecules, 28, 8406 (1995).

12. R. K. McMullan, W. Saenger, J. Fayos, and D. Mootz, Carbohydr. Res., 31, 37 (1973).

13. R. Dodge and W. L. Mattice, Macromolecules, 24, 2709 (1991).

14. Y. Zhan and W. L. Mattice, Macromolecules, 25, 3440 (1992).

15. Y. Zhan and W. L. Mattice, Macromolecules, 25, 4078 (1992).

16. Y. Zhan and W. L. Mattice, J. Chem. Phys., 96, 3279 (1992).

17. Y. Zhan and W. L. Mattice, Macromolecules, 27, 7056 (1994).

18. N. Zheng and W. L. Mattice, Acta Polym., 46, 139 (1995).

19. J. Pozuelo, F. Mendicuti, and W. L. Mattice, Macromolecules, 30, 3685 (1997).

20. M. Clark, R. Cramer, III, and N. Van Opdenbosch, J. Comput. Chem., 19, 982 (1989).

21. D. A. Brant and P. J. Flory, J. Am. Chem. Soc., 87, 663 (1965).

22. D. A. Brant and P. J. Flory, J. Am. Chem. Soc., 87, 2791 (1965).

23. K. D. Goebel, W. L. Dimpfl, and D. A. Brant, Macromolecules, 3, 644 (1970).

24. MOPAC-AMI. Included in Sybyl 6.0.

25. J. Pozuelo, J. M. Madrid, F. Mendicuti, and W. L. Mattice, Comput. Theor. Polym. Sci., 6, 125 (1996).

26. A. Abe, T. Hirano, and T. Tsuruta, Macromolecules, 12, 1092 (1979). 